\title{
Effect of brefelamide on growth factor-induced cell proliferation in 1321N1 human astrocytoma cells
}

\author{
Shigeyoshi Honma, Takahiro Shibuya, Takahiro Ishikawa, Sachina Takasaka, Keita Kouno, \\ Makoto Yoshida
}

Faculty of Pharmacy, Takasaki University of Health and Welfare, Japan

Background: Gliomas arising from glial cells are typical tumors of the central nervous system, and they express various growth factors and their corresponding receptors, which together contribute to malignancy. Brefelamide is a novel aromatic amide isolated from Dictyostelium cellular slime molds. We found that brefelamide has a potent growth inhibitory effect in 1321N1 human astrocytoma cells, but not in PC12 cells. In the present study, we examined the mechanism by which brefelamide inhibited the proliferation of 1321N1 human astrocytoma cells.

Methods: Effect of the growth factor on proliferation of 1321N1 human astrocytoma cells or PC12 cells was assessed using the MTT assay. The expression levels of the growth factor and their receptor were quantified by real-time RT-PCR. EGF receptor, ERK, JNK, AKT, and their phosphorylated products were measured by immunoblotting analysis. Secretion of the growth factor were measured by ELISA.

Results: When 1321N1 human astrocytoma cells were cultured in serum-free medium, administration of the growth factor (EGF, HGF or GDNF) increased the proliferation rate of 1321N1 human astrocytoma cells. The growth factor-induced cell proliferation was inhibited by pretreatment with brefelamide. Brefelamide diminished EGF-induced phosphorylation of EGF receptor at Tyr1068, a Grb2 binding site that leads to an activation of Ras/Raf/ERK system, and reduced expression level of EGF receptor. Moreover, brefelamide inhibited EGF-induced phosphorylation of ERK, HGF-induced phosphorylation of ERK and AKT, and GDNF-induced ERK, AKT and JNK. mRNA expression of HGF receptor and GDNF receptor were inhibited by a pretreatment of brefelamide. Secretion of the HGF and GDNF were reduced by brefelamide.

Conclusions: Brefelamide reduces the proliferation of 1321N1 human astrocytoma cells through multiple effects including reductions of the growth factor receptor expression and secretion, and inhibition of ERK, AKT, and JNK phosphorylation. Brefelamide may represent an effective treatment option for the therapy of gliomas. 